\title{
Skin and gastrointestinal symptoms in COVID-19
}

\author{
Piotr Ciechanowicz ${ }^{1}$, Konrad Lewandowski², Elżbieta Szymańska ${ }^{1}$, Magdalena Kaniewska², \\ Grażyna M. Rydzewska², Irena Walecka ${ }^{1}$
}

${ }^{1}$ Dermatology Department, Centre of Postgraduate Medical Education/Central Clinical Hospital of the Ministry of the Interior and Administration, Warsaw, Poland

2Department of Internal Medicine and Gastroenterology with Inflammatory Bowel Disease Unit, Central Clinical Hospital of the Ministry of the Interior and Administration, Warsaw, Poland

Gastroenterology Rev 2020; 15 (4): 301-308

DOI: https://doi.org/10.5114/pg.2020.101558

Key words: SARS-CoV-2, COVID-19, skin lesions, gastrointestinal symptoms.

Address for correspondence: Prof. Irena Walecka MD, PhD, Dermatology Department, Centre of Postgraduate Medical Education/Central Clinical Hospital of the Ministry of the Interior and Administration, Warsaw, Poland, e-mail: irena.walecka@cskmswia.pl

\begin{abstract}
Introduction: The first cases of coronavirus disease 2019 (COVID-19) were noted in December 2019 in Wuhan province, China. The World Health Organisation (WHO) announced the pandemic status on March 11, 2020. The manifestations of the disease are as follows: fever, cough, fatigue, anosmia and ageusia, dyspnoea, chest pain, muscle soreness, chills, sore throat, rhinitis, headache, gastrointestinal (GI) symptoms, and dermal lesions.

Aim: To evaluate the relationship between dermal lesions and GI symptoms in a group of COVID-19 patients.

Material and methods: A group of 441 COVID-19 patients admitted to the Central Clinical Hospital of the Ministry of the Interior and Administration in Warsaw between March $15^{\text {th }}$ and June $15^{\text {th }}, 2020$.

Results: Of 441 patients with confirmed SARS-CoV-2 infection, 255 (58.5\%) experienced gastrointestinal (GI) symptoms: lack of appetite was reported in 124 (48.6\%) cases, diarrhoea was noted in 109 (42.7\%), abdominal pain in 95 (37.3\%), vomiting in 37 (14.5\%), and nausea in $32(12.5 \%)$ cases. Eight (1.81\%) patients had dermal lesions: erythematous macular lesions (2 patients $-25 \%)$, erythematous infiltrated lesions (2; 25\%), erythematous infiltrated and exfoliative lesions (3; $37.5 \%)$, erythematous papular lesions (3; 37.5\%), and erythematous oedematous lesions $(2 ; 25 \%)$. All of those patients reported gastrointestinal symptoms during the hospitalisation.

Conclusions: The following study analyses possible causes of dermal lesions and their coexistence with Gl symptoms. Several possible theories were taken into account, including the microbiota alterations and issue of drug-related complications.
\end{abstract}

\section{Introduction}

The first cases of new severe acute respiratory syndrome coronavirus 2 (SARS-CoV-2), a pathogen that triggers the symptoms of coronavirus disease 2019 (COVID-19), were noted in December 2019 in Wuhan province, China. The rapid spread of the disease worldwide was the reason why the World Health Organisation (WHO) announced the pandemic status on March 11,2020 [1]. The incubation of the virus lasts from 2 to 14 days, with the most common manifestations being fever, cough, fatigue, anosmia, and ageusia. Moreover, patients may report dyspnoea, chest pain, muscle soreness, chills, sore throat, rhinitis, and headache [2, 3].

Gastrointestinal (GI) symptoms were also described in the course of COVID-19, e.g. stomach pain, diarrhoea, nausea, vomiting, and lack of appetite [4-9]. The major- ity of cases of SARS-CoV-2 infection have an asymptomatic or paucisymptomatic course and subside without treatment $[10,11]$. However, persistent manifestations that do not subside with the use of anti-inflammatory and antipyretic drugs, exacerbating dyspnoea and the aggravation of the general condition of the patient, may require the introduction of specialist treatment, sometimes in intensive care units $[12,13]$.

Additionally, research conducted worldwide showed an increasing number of patients infected with SARSCoV-2 who developed dermal lesions. The most commonly described dermal manifestations included chilblain-like and vesicular lesions, urticaria, maculopapular lesions, livedo reticularis-like, or necrotic lesions [14]. Moreover, dermal lesions may be the only manifestations of COVID-19, particularly in children, in whom both 
the morphology and the incidence of individual dermatoses is different from the general population [15]. Several less common dermatoses occur apart from the above described dermal lesions observed in COVID-19 patients, with the aetiology mostly associated with the disease or the reaction of the body to SARS-CoV-2 infection. The occurrence of those dermatoses may be linked to the use of drugs in COVID-19 therapy [16-18].

\section{Aim}

The aim of the study was to evaluate the relationship between dermal lesions and GI symptoms in a group of 441 COVID-19 patients admitted to the Central Clinical Hospital of the Ministry of Interior and Administration. A total of 255 (57.8\%) patients had Gl symptoms, of whom 8 (1.81\%) had dermal lesions.

\section{Material and methods}

After the WHO declared a pandemic, the Central Clinical Hospital of the Ministry of the Interior and Administration in Warsaw was transformed into an infectious disease hospital. Between March $15^{\text {th }}$ and June $15^{\text {th }}, 2020,441$ patients with a confirmed SARSCoV-2 infection were hospitalised. Of the entire cohort, 255 (57.8\%) presented Gl symptoms. The most common symptom was the lack of appetite, which was reported in 124 (48.6\%) cases. Other GI symptoms, like diarrhoea, were noted in 109 (42.7\%), abdominal pain in 95 (37.3\%), vomiting in 37 (14.5\%), and nausea in $32(12.5 \%)$ cases (Table I). In the group with GI symptoms, a few patients also had skin lesions.

Until recently, the Food and Drug Administration has not approved any drug for COVID-19 treatment, but numerous drugs used for other indications, also including new preparations, proved to be effective in the reduction of the manifestations of the disease $[19,20]$. Their effectiveness and safety are under research in numerous clinical trials worldwide [21]. Drugs recommended

Table I. Prevalence of individual gastrointestinal (GI) symptoms in COVID-19 patients (cases) $(n=441)$

\begin{tabular}{lc} 
Parameter & $n(\%)$ \\
\hline Gastrointestinal symptoms: & $255(57.8)$ \\
\hline Abdominal pain & $95(37.3)$ \\
\hline Diarrhoea & $109(42.7)$ \\
\hline Nausea & $32(12.5)$ \\
\hline Vomiting & $37(14.5)$ \\
\hline Lack of appetite & $124(48.6)$ \\
\hline No gastrointestinal symptoms & $186(42.2)$
\end{tabular}

by the National Institutes of Health, which demonstrated some effectiveness in the treatment of patients with COVID-19, include the following [20, 22]:

- antimalarial preparations - chloroquine and hydroxychloroquine;

- azithromycin (in case of pneumonia, most commonly combined with antimalarial preparations);

- HIV protease inhibitors - Lopinavir/Ritonavir;

- RNA polymerase inhibitor - Remdesivir;

- glucocorticosteroid preparations - dexamethasone was shown to reduce mortality in patients requiring oxygen therapy.

An increasing amount of research is conducted by medical centres worldwide to assess the effectiveness of the plasma of "convalescents" in the treatment of SARS-COV-2 infection. Numerous clinical trials confirmed the effectiveness of this modality. However, the number of studies and treated patients is still too low to draw unambiguous conclusions regarding the effectiveness and safety of the use of plasma in the treatment of COVID-19 [23, 24].

\section{Results}

Eight patients of the Central Clinical Hospital of the Ministry of the Interior and Administration in Warsaw were reported to present dermal lesions associated with SARS-CoV-2 infection. The lesions included: erythematous macular lesions (2 patients $-25 \%$ ), erythematous infiltrated lesions (2; 25\%), erythematous infiltrated and exfoliative lesions ( $3 ; 37.5 \%)$, erythematous papular lesions ( $3 ; 37.5 \%)$, and erythematous oedematous lesions (2; $25 \%)$. What is remarkable, all of those patients reported gastrointestinal symptoms during the hospitalisation. Additionally, in 4 of them, the clinical picture and the histopathological results of derma lesion examination indicated that dermatoses and the gastrointestinal problems seem to be a drug-related reaction following the treatment of COVID-19.

The first described patient was a 74-year-old man with a history of dilated cardiomyopathy and chronic coronary syndrome. The patient was hospitalised because of an episode of fainting and a fall preceded by chest pain, headache, exacerbation of chronic dyspnoea, and high values of arterial blood pressure (up to $230 / 120 \mathrm{~mm} \mathrm{Hg}$ ). During the hospitalisation in the District Hospital in Sochaczew, the patient underwent right-sided decompression of post-traumatic pneumothorax and was administered antibiotic treatment. The patient was transferred to continue the treatment in the Central Clinical Hospital of the Ministry of the Interior and Administration in Warsaw due to the confirmation of SARS-CoV-2 infection (a positive RT-PCR test result on April $\left.18^{\text {th }}, 2020\right)$. On admission no dermal lesions or 
gastrointestinal manifestations were present. Due to the fact that the patient had undergone pneumothorax decompression and had right-sided pneumonia in the course of COVID-19, he was consequentively administered oral amoxicillin and clavulanic acid, levofloxacin, azithromycin, trimethoprim, and sulfamethoxazole. On April $21^{\text {st }}, 2020$ the patient developed erythematous and erythematous macular lesions accompanied by pruritus in the gluteal area (Figure 1). He was administered parenteral clemastinum and topical clobetasol. Azithromycin and levofloxacin were discontinued. The dermal lesions gradually subsided and disappeared over a few days. Histopathological examination revealed a minor acanthotic hyperplasia of the epidermis and moderately intensified mixed inflammatory infiltration located around the vessels in the papillary dermis, locally diffuse in the epidermis. Moreover, two microabscesses were confirmed, together with the features of the stimulation and degeneration of the basal layer. The picture might correspond with lesions attributable to drug use. During the stay, the patient reported epigastric abdominal pain, so he received a proton pump inhibitor (PPI). The symptoms improved during the following days of hospitalisation.

Another described case was an 83-year-old man hospitalised due to the exacerbation of chronic obstructive pulmonary disease (body temperature over $38^{\circ} \mathrm{C}$, non-productive cough) and SARS-CoV- 2 infection (a positive RT-PCR test result obtained on April $16^{\text {th }}, 2020$ ). On admission no dermal lesions was present. On day 4 after introducing ceftazidime (because of fever and the picture of ground glass opacity visible in the computed tomography of the chest) the patient developed diffuse erythematous infiltrated lesions within the trunk and limbs with the greatest severity on the back and buttocks (Figure 2). The dermal lesions were accompanied

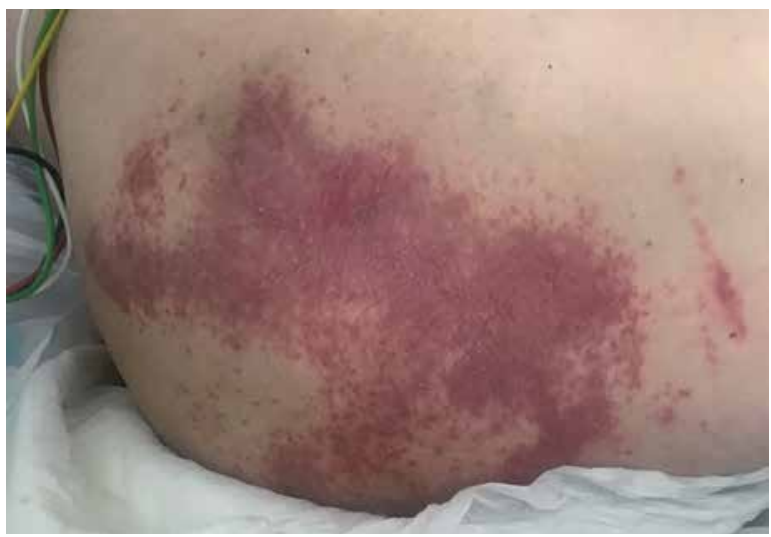

Figure 1. Erythematous and erythematous macular lesions accompanied by pruritus in the gluteal area by pruritus. The use of parenteral clemastinum and local clobetasol, and the discontinuation of ceftazidime in further treatment resulted in the marked reduction of dermal lesions and pruritus. Histopathological examination revealed lesions within the epidermis characterised by local acanthosis and atrophy. Hyperkeratotic lesions were described on the surface of the mass, while elastosis - the features of skin atrophy - were seen in the stromal layer. Numerous thin-walled vessels and perivascular inflammatory infiltrations of mononuclear cells and eosinophils were present subepidermally. The microscopic picture was suggestive of atrophic lesions, which may have been drug-induced. The patient was taking a proton pump inhibitor (PPI) due to dyspepsia. During his hospital stay, he was taking chloroquine and azithromycin from the start of his hospitalisation. He developed diarrhoea (defined as > 3 loose bowel movements) on the third day of hospitalisation and was diagnosed with CDI. The patient had CDI risk factors: age, hospitalisation, presence of comorbidities, taking PPI, and antibiotic therapy. Vancomycin $250 \mathrm{mg}$ administered orally every $6 \mathrm{~h}$ was included in the treatment [25].

Next described clinical case was an 86-year-old woman with a history of hypertension, brain injury of vascular origin, and two myocardial infarctions. The patient was referred by the District Hospital in Sochaczew and admitted to the Central Clinical Hospital of the Ministry of the Interior and Administration in Warsaw due to a confirmed SARS-CoV-2 infection (a positive RT-PCR test result obtained on April $20^{\text {th }}, 2020$ ) and the symptoms of a urinary tract infection. On admission the patient presented no dermal lesions or gastrointestinal symptoms. On admission the patient also underwent chest radiography, which showed streaky hilar opacities on the right side, and in the inferior region of the left lung. Initially, the treatment included parenteral azith-

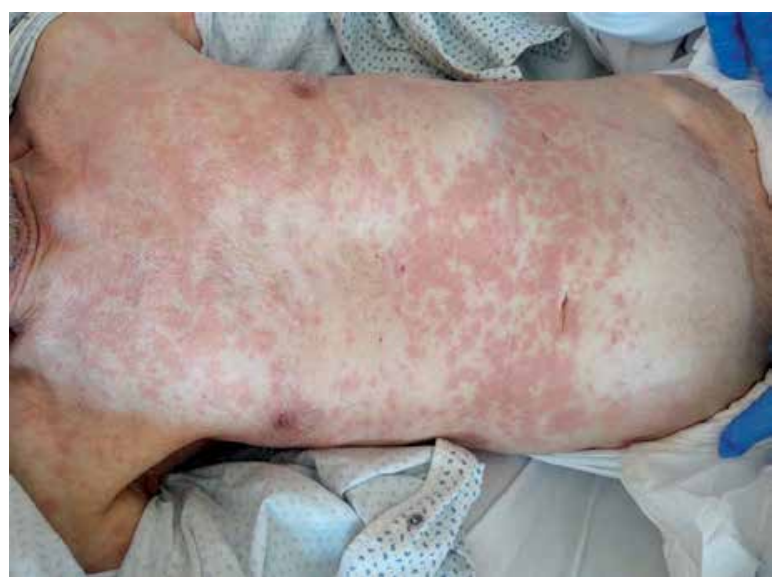

Figure 2. Diffuse erythematous infiltrated lesions within the trunk 
romycin, chloroquine, and subcutaneous low-molecular-weight heparin - drugs which the patient was receiving on a regular basis - and oxygen therapy via nasal cannula. As the parameters of inflammation increased, antibiotic therapy was modified, and the following drugs were introduced consequentively: piperacillin, tazobactam, and ciprofloxacin, followed by imipenem, cilastatin, lopinavir, and ritonavir. On May $10^{\text {th }}, 2020$ the patient developed erythematous infiltrated and exfoliative lesions elevated circumferentially, scarce macules on the skin of the back and abdomen, and erythematous and exfoliative lesions within the right forearm and the left orbital area (Figures $3 \mathrm{~A}, \mathrm{~B}$ ). The treatment included oral cetirizine and local clobetasol. The preparations of ciprofloxacin and imipenem/cilastatin were discontinued. The dermal lesions gradually subsided and disappeared after several days. Histopathological examination revealed non-specific inflammatory lesions whose drug-induced aetiology could not be ruled out: atrophic epidermis with the features of spongiosis, and a solitary microabscess subcorneally. Oedema was visible in the dermis with a moderate mixed inflammatory response (lymphocytes and neutrophils) with the inflammatory cells infiltrating the epidermis. She was taking a PPI for gastroesophageal reflux disease (GERD). During her stay, on the sixth day of hospitalisation, she developed diarrhoea and was diagnosed with CDI. Vancomycin $250 \mathrm{mg}$ administered orally every $6 \mathrm{~h}$ was included in the treatment. The patient had CDI risk factors: age, hospitalisation, concomitant diseases, and the use of antibiotics [25].

A 74-year-old woman with diffuse large B-cell lymphoma is an example of dermal lesions concomitant with gastrointestinal symptoms in the course of COVID-19. The patient was undergoing chemothera- py at the time of the admission. She had a history of acute kidney injury in 2019 and recurrent left-sided parotid sialadenitis. The patient was admitted to the Central Clinical Hospital of the Ministry of the Interior and Administration in Warsaw on an emergency basis due to SARS-CoV-2 infection (a positive RT-PCR test result obtained on April 15th 2020$)$. Drug history included a course of chemotherapy administered on April $2^{\text {nd }}$, 2020. A week later the patient developed fever. She was treated with meropenem, and then amikacin was administered. On admission the patient had disseminated erythematous and papular lesions on the edges of erythematous lesions on the trunk and limbs accompanied by pruritus. Treatment with clemastinum was introduced, and amikacin was discontinued. The dermal lesions subsided over a few days. Histopathological examination revealed a fragment of the skin covered with thinned epidermis with the features of hyperkeratosis. Moreover, the fibrous stromal layer included solitary thin-walled vessels and scarce lymphocytes around them. The patient was taking a PPI due to dyspepsia, which had been diagnosed prior to admission to the hospital. During her stay, she received antibiotics other than azithromycin and chloroquine. On the fourth day, she developed diarrhoea, but no Clostridioides difficile was detected in the stools.

\section{Discussion}

Dermal lesions and gastrointestinal problems may both be related to SARS-CoV-2 infection. However, in some patients, manifestations including dermatoses, nausea, vomiting, diarrhoea, abdominal pain, and lack of appetite may be associated with the drugs used in the therapy of COVID-19.
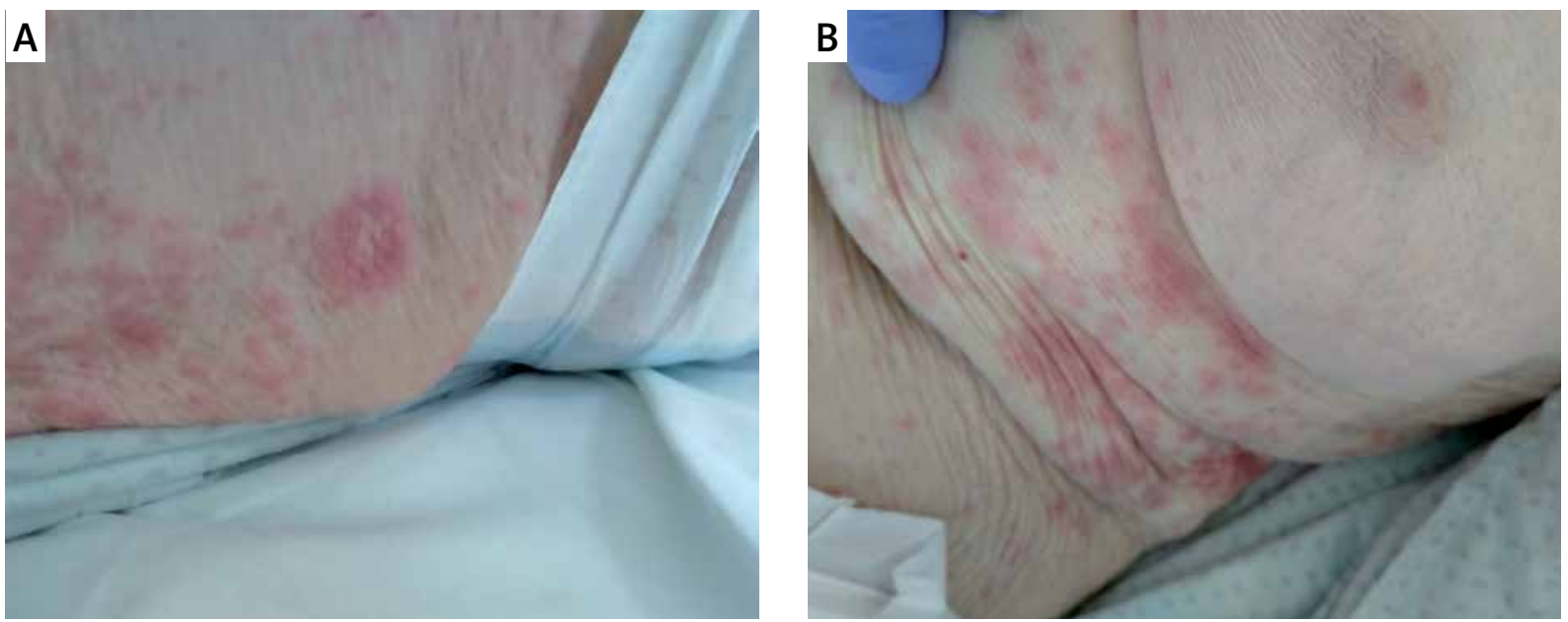

Figure 3. A - Erythematous infiltrated and exfoliative lesions elevated circumferentially, B - scarce macules on the skin of the abdomen 
Scientific reports indicate a strong relationship between the occurrence of GI symptoms and COVID-19 [9]. The causes of their occurrence are still unclear. The presence of genetic material in stool samples in people infected with SARS-CoV-2 seems to be a promising explanation [9, 26-29]. SARS-CoV-2 has a high affinity for the angiotensin II converting enzyme (ACE2) receptor, which is located in various tissues, including the epithelial cells of the GI tract $[30,31]$. SARS-CoV-2 virus RNA was isolated from gastrointestinal epithelial cells, confirming the hypothesis of a gastrointestinal infection. Such an infection is likely to cause dysfunction of the ileum and colon, subsequently leading to various gastrointestinal symptoms seen in COVID-19 patients [27]

The skin constitutes a barrier protecting the body from the penetration by various pathogens: bacteria, fungi, and even viruses. Xue et al. presented the results of their research, which showed a high expression of ACE2 in keratinocytes, particularly those located in the stratum basale [32]. This is associated with the risk of a virus penetrating into the body through the skin (percutaneous infection), especially in individuals using protective clothing and skin disinfectants that damage the epidermal barrier (e.g. in medical personnel) [33]. Furthermore, keratinocytes may be the target of SARSCoV-2 spreading with the blood in patients with fully symptomatic COVID-19 [34].

Manalo et al. presented two possible mechanisms of developing livedo-like lesions in individuals infected with SARS-CoV-2. According to the authors, such dermal lesions were associated with DIC and macrothromboses in patients with a more severe course of the disease, while in milder cases of COVID-19 the microthrombus formations created by inflammatory cytokines or ACE2 entry into cells might be responsible for the occurrence of livedo reticularis [35].

Several viruses from the Coronaviridae family, e.g. severe acute respiratory syndrome coronavirus (SARS(OV) and Middle East respiratory syndrome coronavirus (MERS-CoV), could cause gastroenteritis [36-38]. The presence of human enterocyte involvement in SARS-CoV-2 infections was also suggested by in vitro data [39].

Despite numerous similarities between manifestations induced by SARS-CoV-2 and other coronaviruses responsible for previous epidemics, no dermal lesions were confirmed to be related to SARS-CoV or MERS-CoV infections [40].

Another factor that may be responsible for GI symptoms is the "cytokine storm" that occurs in COVID-19. IL-6, IL-8, and IL-10 cytokines, the elevated levels of which were correlated with the severity of COVID-19, especially in critically ill patients, were associated with
ICU admission, respiratory failure, and poor prognosis [41-43]. The "cytokine storm" results from the acutely increased levels of pro-inflammatory cytokines including IL-6, IL-1, TNF- $\alpha$, and interferon. Such an increase in cytokines results in the influx of various immune cells, including macrophages, neutrophils, and T cells, which causes damage of the vascular barrier, capillary damage, enterocyte and diffuse alveolar damage, and multiorgan failure [44].

The pathomechanism of dermal lesions has not been fully elucidated in the course of SARS-CoV-2 infection. Urticaria and erythematous papular eruptions seemed to be induced by drugs used in the treatment of COVID-19. They might also result from "cytokine storm" $[45,46]$.

Vascular dermatoses in the form of acute ischaemic lesions were initially described in adult patients with severe SARS-CoV-2 infection [47]. The above changes probably resulted from clotting disorders and were associated with microthrombus formation, leading to pulmonary embolism, strokes, and disseminated intravascular coagulation [48-51]. According to Magro et al., ecchymosis- or purpura-like lesions are linked to pauci-inflammatory thrombogenic vasculopathy with the extensive deposition of complement components C5b-9 and $\mathrm{C} 4 \mathrm{~d}$ within the cutaneous microvasculature of both lesional and non-lesional skin [52]. However, such dermatoses may also be due to adverse drug reactions in the treatment of COVID-19 [46].

However, recently, an increasing number of paediatricians, particularly those from regions most affected by COVID-19, have reported the occurrence of chilblain-like dermatoses among patients who had never experienced perniosis or Raynaud symptoms [53-55].

According to the literature, numerous mechanisms were presented to explain the mechanisms responsible for the development of chilblain-like lesions, e.g. "immune dysregulation, vasculitis, vessel thrombosis, or neoangiogenesis" [56].

Kolivras et al. [57] presented the histopathological picture of chilblain skin lesions, in which they described papillary dermal oedema with perivascular and perieccrine lymphocytic infiltration along with scattered necrotic keratinocytes in the superficial layers of the epidermis. Microangiopathic changes may be caused by an increased reaction associated with the increased production of interferon type 1, which occurs during SARS-COV-2 infection, especially in children and young adults. However, it may be a protective factor against the occurrence of "cytokine storm" and full-blown COVID-19 [57-59].

Gastrointestinal symptoms and/or skin lesions could be connected with microbiota alterations. Zuo 
et al. investigated changes in faecal microbiomes of patients with COVID-19 during hospitalisation and estimated the associations with the severity and faecal shedding of SARS-CoV-2 [60]. They performed shotgun metagenomic sequencing analyses of faecal samples obtained from 15 patients with COVID-19. The samples were collected 2 or 3 times per week during hospitalisation. Patients with SARS-CoV-2 infection had significant alterations in the faecal microbiomes compared to the controls, characterised by the enrichment of opportunistic pathogens and the depletion of beneficial commensals, at the time of hospitalisation and at all time points during hospitalisation. Depleted symbionts and gut dysbiosis persisted even after the clearance of SARS CoV-2. The baseline abundance of Coprobacillus, Clostridium ramosum, and Clostridium hathewayi correlated with COVID-19 severity; there was an inverse correlation between the abundance of Faecalibacterium prausnitzii (an anti-inflammatory bacterium) and disease severity. Over the course of hospitalisation, Bacteroides dorei, Bacteroides thetaiotaomicron, Bacteroides massiliensis, and Bacteroides ovatus, which downregulate the expression of angiotensin-converting enzyme 2 in the murine gut, correlated inversely with SARS-CoV-2 load in the faecal samples of patients. They found persistent alterations in the faecal microbiome during the time of hospitalisation, compared to the controls. Faecal microbiota alterations were associated with the faecal levels of SARS-CoV-2 and COVID-19 severity [60].

\section{Conclusions}

In this paper we wanted to emphasise the significance of taking a detailed history concerning drug hypersensitivity and the necessity of implementing suitable prophylaxis when administering antibiotics in order to avoid Clostridium difficile infection. Half of the dermal lesions in the entire cohort were associated with drug-induced lesions during the treatment of SARS-CoV-2 infection. Thus, from the analysis of the observed cases, no conclusion can be drawn about the aetiopathogenetic dependence of skin lesions and $a b$ dominal symptoms, although changes in the intestinal microbiota can be taken into account each time.

\section{Conflict of interest}

The authors declare no conflict of interest.

\section{References}

1. World Health Organization. Novel Coronavirus Situation Reports 2020. Available from: https://covid19.who.int.

2. Jiang F, Deng L, Zhang L, et al. Review of the clinical characteristics of coronavirus disease 2019 (COVID-19). J Gen Intern Med 2020; 35: 1545-9.
3. Mayo Clinic. Coronavirus disease 2019 (COVID-19) 2020. Available at: https://www.mayoclinic.org/diseases-conditions/coronavirus/symptoms-causes/syc-20479963.

4. Ramachandran P, Onukogu I, Ghanta S, et al. Gastrointestinal symptoms and outcomes in hospitalized coronavirus disease 2019 patients. Dig Dis 2020; 38: 373-9.

5. Mandal A, Konala VM, Adapa S, et al. Gastrointestinal manifestations in COVID-19 infection and its practical applications. Cureus 2020; 12: e8750.

6. Ouali SE, Achkar JP, Lashner B, Regueiro M. Gastrointestinal manifestations of COVID-19. Cleve Clin J Med 2020; doi:10.3949/ccjm.87a.ccc049.

7. Gupta A, Madhavan MV, Sehgal K, et al. Extrapulmonary manifestations of COVID-19. Nat Med 2020; 26: 1017-32.

8. Rokkas T. Gastrointestinal involvement in COVID-19: a systematic review and meta-analysis. Ann Gastroenterol 2020; 33: 355-65.

9. Cheung KS, Hung IFN, Chan PPY, et al. Gastrointestinal manifestations of SARS-CoV-2 infection and virus load in fecal samples from a hong kong cohort: systematic review and meta-analysis. Gastroenterology 2020; 159: 81-95.

10. Chen P, Zhang Y, Wen Y, et al. Clinical and demographic characteristics of cluster cases and sporadic cases of coronavirus disease 2019 (COVID-19) in 141 patients in the Main District of Chongqing, China, between January and February 2020. Med Sci Monit 2020; 26: e923985.

11. Nishiura H, Kobayashi T, Miyama T, et al. Estimation of the asymptomatic ratio of novel coronavirus infections (COVID-19). Int J Infect Dis 2020; 94: 154-5.

12. Kumar A, Arora A, Sharma P, et al. Clinical features of COVID-19 and factors associated with severe clinical course: a systematic review and meta-analysis. SSRN 2020; 21: 3566166.

13. Hunt M, Koziatek C. A case of COVID-19 pneumonia in a young male with full body rash as a presenting symptom. Clin Pract Cases Emerg Med 2020; 4: 219-21.

14. Ciechanowicz P, Szymańska E, Wiszniewski K, Walecka I. Cutaneous manifestations of coronavirus disease 2019 - review of literature and case reports. Adv Dermatol Allergol 2020. doi: https://doi.org/10.5114/ada.2020.95958.

15. Ciechanowicz P, Dopytalska K, Szczerba M, et al. Dermatological manifestations of the Coronavirus disease 2019 in children: a systemic review. Adv Dermatol Allergol 2020. doi: https:// doi.org/10.5114/ada.2020.99294.

16. Jimenez-Cauhe J, Ortega-Quijano D, Prieto-Barrios M, et al. Reply to "COVID-19 can present with a rash and be mistaken for dengue": petechial rash in a patient with COVID-19 infection. J Am Acad Dermatol 2020; 83: e141-2.

17. Mahe A, Birckel E, Krieger S, et al. A distinctive skin rash associated with coronavirus disease 2019? J Eur Acad Dermatol Venereol 2020; 34: e246-7.

18. Guan WJ, Ni ZY, Hu Y, et al. Clinical characteristics of coronavirus disease 2019 in China. N Engl J Med 2020; 382: 1708-20.

19. U.S. Food and Drug Administration. [Internet] Coronavirus (COVID-19). Drugs 2020 [updated July 8, 2020]. Available from: https://www.fda.gov/drugs/emergency-preparedness-drugs/ coronavirus-covid-19-drugs.

20. National Institutes of Health. Potential Antiviral Drugs Under Evaluation for the Treatment of COVID-19 2020. Updated July 
24, 2020. Available from: https://www.covid19treatmentguidelines.nih.gov/antiviral-therapy/.

21. U.S. National Library of Medicine: ClinicalTrials.gov [Internet] COVID-19 Studies from the World Health Organization Database [updated July 29, 2020]. Available from: https://clinicaltrials.gov/ct2/results?cond=COVID-19.

22. Sanders JM, Monogue ML, Jodlowski TZ, Cutrell JB. Pharmacologic treatments for coronavirus disease 2019 (COVID-19) a review. JAMA 2020; 323: 1824-36.

23. Li L, Zhang W, Hu Y, et al. Effect of convalescent plasma therapy on time to clinical improvement in patients with severe and life-threatening COVID-19: a randomized clinical trial. JAMA 2020; 324: 460-70.

24. Joyner MJ, Wright RS, Fairweather D, et al. Early safety indicators of COVID-19 convalescent plasma in 5,000 patients. J Clin Invest 2020; 130: 4791-7.

25. Kukla M, Adrych K, Dobrowolska A, et al. Guidelines for Clostridium difficile infection in adults. Gastroenterology Rev 2020 15: 1-21.

26. Young BE, Ong SWX, Kalimuddin S, et al. Epidemiologic features and clinical course of patients infected with SARS-CoV-2 in Singapore. JAMA 2020; 323: 1488-94.

27. Xiao F, Tang M, Zheng X, et al. Evidence for gastrointestinal infection of SARS-CoV-2. Gastroenterology 2020; 158: 1831-3 e3.

28. Holshue ML, DeBolt C, Lindquist S, et al. First case of 2019 novel coronavirus in the United States. N Engl J Med 2020; 382: 929-36.

29. Wu Y, Guo C, Tang L, et al. Prolonged presence of SARS-CoV-2 viral RNA in faecal samples. Lancet Gastroenterol Hepatol 2020; 5: 434-5.

30. Du M, Cai G, Chen F, et al. Multiomics evaluation of gastrointestinal and other clinical characteristics of COVID-19. Gastroenterology 2020; 158: 2298-301.

31. Zhang $\mathrm{H}$, Kang Z, Gong $\mathrm{H}$, et al. The digestive system is a potential route of 2019-nCov infection: a bioinformatics analysis based on single-cell transcriptomes. BioRxiv 2020. doi: https:// doi.org/10.1101/2020.01.30.927806.

32. Xue X, Mi Z, Wang Z, et al. High expression of ACE2 on keratinocytes reveals skin as a potential target for SARS-CoV-2. J Invest Dermatol 2020. doi: 10.1016/j.jid.2020.05.087.

33. Yan Y, Chen H, Chen L, et al. Consensus of Chinese experts on protection of skin and mucous membrane barrier for healthcare workers fighting against coronavirus disease 2019. Dermatol Ther 2020; 33: e13310.

34. To KK, Tsang OT, Leung WS, et al. Temporal profiles of viral load in posterior oropharyngeal saliva samples and serum antibody responses during infection by SARS-CoV-2: an observational cohort study. Lancet Infect Dis 2020; 20: 565-74.

35. Manalo IF, Smith MK, Cheeley J, Jacobs R. A dermatologic manifestation of COVID-19: Transient livedo reticularis. J Am Acad Dermatol 2020; 83: 700 .

36. Leung WK, To KF, Chan PK, et al. Enteric involvement of severe acute respiratory syndrome-associated coronavirus infection. Gastroenterology 2003; 125: 1011-7.

37. Chan KH, Poon LL, Cheng VC, et al. Detection of SARS coronavirus in patients with suspected SARS. Emerg Infect Dis 2004; 10: 294-9.
38. Assiri A, Al-Tawfiq JA, Al-Rabeeah AA, et al. Epidemiological, demographic, and clinical characteristics of 47 cases of Middle East respiratory syndrome coronavirus disease from Saudi Arabia: a descriptive study. Lancet Infect Dis 2013; 13: 752-61.

39. Lamers MM, Beumer J, van der Vaart J, et al. SARS-CoV-2 productively infects human gut enterocytes. Science 2020; 369: 50-4.

40. Singh H, Kaur H, Singh K, Sen CK. Cutaneous manifestations of COVID-19: a systematic review. Adv Wound Care 2020. doi: 10.1089/wound.2020.1309.

41. Xu X, Yu MQ, Shen Q, et al. Analysis of inflammatory parameters and disease severity for 88 hospitalized COVID-19 patients in Wuhan, China. Int J Med Sci 2020; 17: 2052-62.

42. Akbari $H$, Tabrizi R, Lankarani KB, et al. The role of cytokine profile and lymphocyte subsets in the severity of coronavirus disease 2019 (COVID-19): a systematic review and meta-analysis. Life Sci 2020; 258: 118167.

43. Del Valle DM, Kim-Schulze S, Hsin-Hui H, et al. An inflammatory cytokine signature helps predict COVID-19 severity and death. medRxiv 2020. doi: https://doi.org/10.1101/2020.05. 28.20115758

44. Ragab D, Salah Eldin H, Taeimah M, et al. The COVID-19 cytokine storm; what we know so far. Front Immunol 2020; 11 : 1446.

45. Herrero-Moyano M, Capusan TM, Andreu-Barasoain M, et al. A clinicopathological study of eight patients with COVID-19 pneumonia and a late-onset exanthema. J Eur Acad Dermatol Venereol 2020; doi: 10.1111/jdv.16631.

46. Tursen U, Tursen B, Lotti T. Cutaneous side-effects of the potential COVID-19 drugs. Dermatol Ther 2020; 33: e13476.

47. Zhang Y, Cao W, Xiao M, et al. Clinical and coagulation characteristics in 7 patients with critical COVID-2019 pneumonia and acro-ischemia. Zhonghua Xue Ye Xue Za Zhi 2020; 41: 302-7.

48. Llamas-Velasco M, Munoz-Hernandez P, Lazaro-Gonzalez J, et al. Thrombotic occlusive vasculopathy in skin biopsy from a livedoid lesion of a COVID-19 patient. Br J Dermatol 2020. doi: 10.1111/bjd.19222.

49. Labe P, Ly A, Sin C, et al. Erythema multiforme and Kawasaki disease associated with COVID-19 infection in children. J Eur Acad Dermatol Venereol 2020; 34: e539-41.

50. Danzi GB, Loffi M, Galeazzi G, Gherbesi E. Acute pulmonary embolism and COVID-19 pneumonia: a random association? Eur Heart J 2020; 41: 1858.

51. Tang N, Li D, Wang X, Sun Z. Abnormal coagulation parameters are associated with poor prognosis in patients with novel coronavirus pneumonia. J Thromb Haemost 2020; 18: 844-7.

52. Magro C, Mulvey JJ, Berlin D, et al. Complement associated microvascular injury and thrombosis in the pathogenesis of severe COVID-19 infection: a report of five cases. Transl Res 2020; 220: 1-13.

53. Colonna C, Monzani NA, Rocchi A, et al. Chilblain-like lesions in children following suspected COVID-19 infection. Pediatr Dermatol 2020; 37: 437-40.

54. Garcia-Lara G, Linares-Gonzalez L, Rodenas-Herranz T, Ruiz-Villaverde R. Chilblain-like lesions in pediatrics dermatological outpatients during the COVID-19 outbreak. Dermatol Ther 2020; e13516. 
55. Ramondetta A, Panzone M, Dapavo P, et al. Chilblain acral lesions in the COVID-19 era. Are they marker of infection in asymptomatic patients? J Eur Acad Dermatol Venereol 2020. doi: 10.1111/jdv.16636.

56. Bouaziz JD, Duong T, Jachiet $M$, et al. Vascular skin symptoms in COVID-19: a french observational study. J Eur Acad Dermatol Venereol 2020. doi: 10.1111/jdv.16544.

57. Kolivras A, Dehavay F, Delplace D, et al. Coronavirus (COVID-19) infection-induced chilblains: a case report with histopathologic findings. JAAD Case Rep 2020; 6: 489-92.

58. Zhou ZR, Li Z, et al. Overly exuberant innate immune response to SARS-CoV-2 infection. SSRN Electronic Journal 2020.

59. Andina D, Noguera-Morel L, Bascuas-Arribas M, et al. Chilblains in children in the setting of COVID-19 pandemic. Pediatr Dermatol 2020; 37: 406-11.

60. Zuo T, Zhang F, Lui GCY, et al. Alterations in gut microbiota of patients with COVID-19 during time of hospitalization. Gastroenterology 2020; 159: 944-55.

Received: 12.11 .2020

Accepted: 20.11 .2020 